\title{
Plants Used as Ethanomedicine by the Adi Tribes of Sille-Oyan Circle of Arunachal Pradesh: Source for Livelihood and Medicine
}

\author{
Tantulung Tatan*, T. S. Mehra, Senjem Semba, \\ Haffis Mohammed and Fullmoon Puwein \\ College of Horticulture and Forestry, Central Agricultural University, Pasighat, \\ Arunachal Pradesh-791102, India \\ *Corresponding author
}

\section{A B S T R A C T}

\section{Keywords}

Arunachal Pradesh, Adi Tribe,

Traditional, Medicinal, Plants

Article Info

\section{Accepted:}

18 November 2020

Available Online:

10 December 2020
The objectives of the present study were to document the ethno-medicinal plants use by the Adi tribes of Sille-Oyan Circle of Arunachal Pradesh for therapeutic diseases. These are not only for treat ailments but also for the potential source of economy of the peoples as well as to the village. The present study recorded 59 plant species which belong to 34 families for various uses from which Asteraceae family, (6) is mainly used and mostly are herbs. The local practitioner has a vast knowledge regarding identification, extraction, uses, preparation and applications of plants and herbs for the treatment of various kinds of ailments locally. Traditional drug treatments are the mainstay of healthcare in this region and are known to support the treatment of many illnesses. Some of the medicinal plants used by aboriginal peoples are Zanthoxylum rhetsa, Acmella oleracea, Clerodendum colebrookianum, Houttuynia cordata, Solanum indicum and Solanum nigrum, etc. The folk use some plants has also been scientifically validated such as the leaves of Clerodendrum colebrookianum (Ongin) are consumed by aboriginal peoples to check high blood pressure and the same is evident by the research that the extract of plant possesses hypotensive properties. In the present paper, emphasis is given to highlight some of the important plants consumed by the Adi tribe of Arunachal Pradesh and their medicinal benefits to serve as a basis for their further scientific validation. The study was done to conserve the importance of traditional knowledge of tribes as these tribal people are rapidly acquiring the modern culture and custom threatening the plants and vanishing the traditional value of the plants.

\section{Introduction}

From the early age, people used various materials from nature to cure their ailments to improve their health. There is only one source of medicinal agent i.e., is nature. Many modern drugs are being isolated from natural sources which are based on their use in traditional medicine. These plant- based traditional medical systems continue to play an essential role in health care, with about $80 \%$ of the world's inhabitants relying mainly on traditional medicines for their primary health care (WHO 2002-2005). Ethnomedicine is defined as the interdisciplinary science of biologically active traditionally observed by man (Holmstedt and Bruhn, 1983). India has vast ethnomedicinal 
knowledge since ancient times. Origin of all such knowledge in India is from the great traditional system of Ayurveda. Arunachal Pradesh with a total geographical area of $83,743 \mathrm{sq} \mathrm{km}$. is the largest state of Northeast India which occupies a major portion of the Eastern Himalaya global biodiversity hotspot harbouring over 500 species of medicinal plants, latitude $26^{\circ} 28^{\prime} \mathrm{N}$ and $29^{\circ} 33^{\prime} \mathrm{N}$ and between $91^{\circ} 31^{\prime} \mathrm{E}$ and $97^{\circ} 30^{\prime} \mathrm{E}$ longitude (Mandal et al., 2002). An ethnomedicinal study takes into account knowledge of the Adi tribes inhabiting in the area, with particular reference to their distribution, language/dialect spoken, food habit, rituals and practices, traditional practices followed in agriculture, house building, art and craft objects made out of plant and their products, etc. An ethnomedicinal study of the area, therefore, aims to cover under its purview the whole of information about plants namely plants used as medicine. The Adi language spoken by the people of this region belongs to the Tibeto-Burman language family (Mandal et al., 2002; Singh 1998; Dutta and Ahmad 1995). Earlier studies published on different districts on Adi tribe include Singh et al., (2008), Singh et al., (2012), Nimasow et al., (2012), Baruah et al., (2013) Sharma and Borthakur (2008), Yumnum and Tripathi (2013) and Singh et al., (2010). Adi people celebrate different festivals like solung, etor and aaran etc., which are essential parts of their socio-cultural life. Ethnomedicine studies depend on comprehensively on indigenous and local knowledge; they face the challenge of adequately bringing evidence from these knowledge systems. The challenge arises not only because unwritten forms of knowledge can be difficult to transpose to into written scientific studies (Raid et al., 2006, Berkes, 2018), but more importantly because scientific studies might decontextualize the information and remove it from the cultural environment that gives it meaning (Stevenson 1996). Festivals reflect the traditions, costumes and lifestyle of the people. The festivals are mainly celebrated for feasts, a good harvest of crop, merriment and for narrating the myths, legends, folklore and mythologies.

\section{Materials and Methods}

The present study was done in the Sille-Oyan Circle Villages which is located in East Siang District of Arunachal Pradesh State, India. The method used for collecting the information is both from primary and secondary sources. The relevant secondary information was collected from both published and unpublished materials available in various government agencies, newspapers, libraries, books, journals and magazine. The ethno-medicinal information was collected by field survey among Adi community of the villager and discussions among the informants in their own local dialogue (Adi) and identifying the Knowledgeable traditional healers known as Miri Abu based on their experience on herbal medicine and interviews were carried out with the identified local people and traditional healers. The questionnaire allowed responses on the plant prescribed, part of the plant used, medicinal uses for each part, mode of preparation (i.e., decoction, paste, powder and juice), a form of usage (either fresh or dried) and additional plants used as ingredients. The information collected was noted in the note book.

\section{Results and Discussion}

There are various types of medicine practiced in the world, with its own beliefs and therapeutics but with one collective purpose: easing of disease. The objective of the study was not only for medicinal used but conservation and management were also included for the sustainable use of the plants (Fig. 1). 
Table.1 Plants used by Adi tribe of Sille-Oyan circle of Arunachal Pradesh

\begin{tabular}{|c|c|c|c|c|c|c|c|}
\hline $\begin{array}{l}\text { Sl. } \\
\text { No. }\end{array}$ & $\begin{array}{l}\text { Common } \\
\text { Name }\end{array}$ & Scientific Name & Family & Used & Habit & Part used & Method \\
\hline 1 & $\begin{array}{l}\text { Namsing } \\
\text { ing }\end{array}$ & Ageratum conyzoides $L$. & Asteraceae & $\begin{array}{l}\text { Leaf Paste Applied } \\
\text { On Cuts } \\
\text { For Blood Clotting }\end{array}$ & Herb & Leaves & Poultices \\
\hline 2 & Kordoi & Averrhoa carambola $L$. & Oxalidaceae & For Treat Jaundice & Tree & Ripe Fruits & Raw \\
\hline 3 & Pasi tirbin & Ardisia solanacea Roxb. & Myrsinaceae & $\begin{array}{l}\text { Antidote For Insect } \\
\text { Poisoning }\end{array}$ & Tree & $\begin{array}{l}\text { Tender } \\
\text { Leaves }\end{array}$ & Paste \\
\hline 4 & Buri & Baccaurea ramiflora Lour. & Phyllanthaceae & Constipation & Tree & Fruits & Raw \\
\hline 5 & $\begin{array}{l}\text { Sising } \\
\text { Bayong }\end{array}$ & Begonia josephii A. DC. & Begoniaceae & $\begin{array}{c}\text { Jaundice, Burning Of } \\
\text { Urine }\end{array}$ & Herb & Shoot, Leaves & $\begin{array}{l}\text { Infusion, } \\
\text { Decoction }\end{array}$ \\
\hline 6 & Taso lapyo & Bidens pilosa $L$. & Asteraceae & $\begin{array}{c}\text { Wound-Root } \\
\text { Diarrhoea-Leaves }\end{array}$ & Herb & $\begin{array}{l}\text { Root Paste } \\
\text { Leaves }\end{array}$ & $\begin{array}{l}\text { Paste } \\
\text { Infusions }\end{array}$ \\
\hline 7 & Kat-Buk & $\begin{array}{c}\text { Bryophyllum } \\
\text { pinnatum (Lam.) Oken }\end{array}$ & Crassulaceae & $\begin{array}{l}\text { Skin Burn And } \\
\text { Fracture }\end{array}$ & Shrub & Leaves & Paste \\
\hline 8 & Ramsing & $\begin{array}{c}\text { Caesalpinia } \\
\text { pulcherrima (L.) Sw. }\end{array}$ & Fabaceae & $\begin{array}{l}\text { Constipation And } \\
\text { Kidney Stones }\end{array}$ & Shrub & Leaves & Infusion \\
\hline 9 & $\begin{array}{l}\text { Akon } \\
\text { Atang }\end{array}$ & $\begin{array}{c}\text { Calotropis gigantea }(L .) \\
\text { W.T.Aiton }\end{array}$ & Apocynaceae & $\begin{array}{l}\text { Sprains, Boils And } \\
\text { Body Pains }\end{array}$ & Shrub & Leaves & Raw \\
\hline 10 & Omri & Carica papaya $L$. & Caricaceae & $\begin{array}{c}\text { Cracks Of Feet, } \\
\text { Gastritis, Malaria }\end{array}$ & Herb & Flower, Fruit & $\begin{array}{l}\text { Decoction, } \\
\text { Infusion, Raw }\end{array}$ \\
\hline 11 & Kiling Kro & Centella asiatica (L.) Urb. & Apiaceae & $\begin{array}{l}\text { Gastritis, Malaria- } \\
\text { Typhoid Jaundice }\end{array}$ & Herb & Leaves & Infusion \\
\hline 12 & Jilmil sak & Chenopodium album $L$. & Amaranthaceae & $\begin{array}{c}\text { Anaemia, Debility, } \\
\text { Eye Tonic }\end{array}$ & Herb & $\begin{array}{c}\text { Seeds } \\
\text {,Leaves, Roots }\end{array}$ & Decoction, Infusion \\
\hline
\end{tabular}




\begin{tabular}{|c|c|c|c|c|c|c|c|}
\hline 13 & Hira Tarpa & Cissampelos pareira $L$. & Menispermaceae & $\begin{array}{l}\text { Antidote For } \\
\text { Snakebite }\end{array}$ & Herb & Tubers & Decoction, Infusion \\
\hline 14 & Ongin & $\begin{array}{c}\text { Clerodendrum } \\
\text { colebrookeanum } \\
\text { Walp }\end{array}$ & Verbenaceae & High Blood Pressure & Shrub & Leaves & $\begin{array}{l}\text { Raw, Decoction, } \\
\text { Infusion }\end{array}$ \\
\hline 15 & $\begin{array}{c}\text { Urom } \\
\text { banggen }\end{array}$ & Costus spp. & Costaceae & $\begin{array}{l}\text { Respiratory Problem, } \\
\text { Urinary Problem }\end{array}$ & Herb & $\begin{array}{l}\text { Leaves And } \\
\text { Stem }\end{array}$ & Decoction, Infusion \\
\hline 16 & $\begin{array}{l}\text { Yakana } \\
\text { Kelodi }\end{array}$ & Curcuma caesia Roxb. & Zingiberaceae & $\begin{array}{c}\text { Diarrhoea, Dysentery } \\
\text { And Wound }\end{array}$ & Shrub & Rhizome & Decoction \\
\hline 17 & Makung & Cucumis sativus L. & Cucurbitaceae & $\begin{array}{c}\text { Acidity, Diabetes, } \\
\text { High Blood Pressure, } \\
\text { Stone, Deworming }\end{array}$ & Shrub & Fruit, Seeds & Raw, Boil \\
\hline 18 & Okolibo & $\begin{array}{l}\text { Deeringia amaranthoides } \\
\text { (Lam.) Merr. }\end{array}$ & Amaranthaceae & Piles, Constipation, & Shrub & Leaves & $\begin{array}{l}\text { Decoction, } \\
\text { Infusion }\end{array}$ \\
\hline 19 & Kekut & $\begin{array}{c}\text { Eleutherococcus } \\
\text { trifoliatus (L.) } \\
\text { S.Y.Hu }\end{array}$ & Araliaceae & $\begin{array}{c}\text { For Improve } \\
\text { Memory, Cough And } \\
\text { Cold }\end{array}$ & Shrub & Bark & $\begin{array}{c}\text { Dried, } \\
\text { Decoction }\end{array}$ \\
\hline 20 & Ritak & $\begin{array}{c}\text { Eryngium } \\
\text { floridanum J.M.Coult. } \\
\text { and Rose }\end{array}$ & Apiaceae & $\begin{array}{l}\text { Cough, Cold, Body } \\
\text { Pain, Headache }\end{array}$ & Herb & Leaves & Paste \\
\hline 21 & Gende & $\begin{array}{c}\text { Gynura crepidioides } \\
\text { Benth. }\end{array}$ & Astereacea & $\begin{array}{l}\text { Gastritis, Stone, } \\
\text { Headaches }\end{array}$ & Herb & Leaves & $\begin{array}{l}\text { Decoction, } \\
\text { Infusion }\end{array}$ \\
\hline 22 & Notke & $\begin{array}{l}\text { Gonostegia hirta } \\
\text { (Hassk.) Miq. }\end{array}$ & Urticaceae & $\begin{array}{l}\text { Antidote For Gandhi } \\
\text { Bug Poisoning }\end{array}$ & Herb & Tender Leave & Decoction, Infusion \\
\hline
\end{tabular}




\begin{tabular}{|c|c|c|c|c|c|c|c|}
\hline 23 & Taki sidik & Gynocardia odorata Roxb. & Achariaceae & $\begin{array}{l}\text { Ant Diabetic, Tooth } \\
\text { Decay }\end{array}$ & Tree & $\begin{array}{l}\text { Bark, Leaves, } \\
\text { Fruits }\end{array}$ & Decoction, Infusion \\
\hline 24 & $\begin{array}{l}\text { Rokpomey } \\
\text { o }\end{array}$ & Gynura nepalensis DC. & Asteraceae & Piles. Appendicitis & Herb & $\begin{array}{c}\text { Leaves } \\
\text { And Flower }\end{array}$ & $\begin{array}{l}\text { Decoction, } \\
\text { Infusion }\end{array}$ \\
\hline 25 & Asi pum & Homonoia riparia Lour. & Euphorbiaceae & $\begin{array}{l}\text { Antidote For Snake } \\
\text { Bite, Skin diseases }\end{array}$ & Shrub & Leave & $\begin{array}{l}\text { Decoction, } \\
\text { Poultice, }\end{array}$ \\
\hline 26 & Loram & $\begin{array}{c}\text { Houttuynia cordata } \\
\text { Thunb. }\end{array}$ & Saururaceae & $\begin{array}{l}\text { Deworming, } \\
\text { Gastritis }\end{array}$ & Herb & Leave & $\begin{array}{l}\text { Decoction, } \\
\text { Infusion }\end{array}$ \\
\hline 27 & $\begin{array}{l}\text { Engin } \\
\text { Taree }\end{array}$ & $\begin{array}{l}\text { Plectranthus } \\
\text { esculentus } \\
\text { N.E.Br. }\end{array}$ & Convolvulceae & $\begin{array}{l}\text { Goitre, Dysentery, } \\
\text { Hypertension, } \\
\text { Constipation }\end{array}$ & Herb & Tender Leave & Decoction, Infusior \\
\hline 28 & Beying & $\begin{array}{c}\text { Lasia spinosa }(L .) \\
\text { Thwaites }\end{array}$ & Araceae & $\begin{array}{c}\text { Dysentery, Liver } \\
\text { Tonic }\end{array}$ & Herb & $\begin{array}{l}\text { Leave } \\
\text {,Stem }\end{array}$ & $\begin{array}{l}\text { Decoction, } \\
\text { Infusion }\end{array}$ \\
\hline 29 & Eki sypiak & $\begin{array}{c}\text { Leucas aspera (Willd.) } \\
\text { Link }\end{array}$ & Lamiaceae. & $\begin{array}{c}\text { Reduce Fever, } \\
\text { Antidote, Headache }\end{array}$ & Herb & $\begin{array}{l}\text { Leaves, } \\
\text { Flower }\end{array}$ & Paste \\
\hline 30 & $\begin{array}{l}\text { Singe } \\
\text { Engin }\end{array}$ & Manihot esculenta Crantz & Euphorbiaceae & Debility, Weakness & Shrub & $\begin{array}{c}\text { Tender } \\
\text { Leaves, Tubers }\end{array}$ & Decoction \\
\hline 31 & Kerala & Momordica charantia L & Cucurbitaceae & $\begin{array}{c}\text { Debility, Stone, } \\
\text { Diabetes }\end{array}$ & Climber & $\begin{array}{l}\text { Leaves, } \\
\text { Fruit, }\end{array}$ & Decoction \\
\hline 32 & Aksap & Mussaenda frondosa $L$. & Rubiaceae & Urinary Problem & Tree & Leaves & Decoction \\
\hline 33 & $\begin{array}{l}\text { Take- } \\
\text { mareng }\end{array}$ & Ocimum gratissimum L. & lamiaceae & Cough, Gastritis & Herb & Leave & $\begin{array}{l}\text { Decoction, } \\
\text { Infusion }\end{array}$ \\
\hline 34 & $\begin{array}{l}\text { Riki } \\
\text { modon }\end{array}$ & $\begin{array}{l}\text { Operculina turpethum } \\
\text { (L.) Silva Manso }\end{array}$ & Convolvulaceae & $\begin{array}{l}\text { Cut, Weakness, } \\
\text { Nerve Disorder, } \\
\text { Jaundice, } \\
\text { Constipation }\end{array}$ & Herb & Whole & $\begin{array}{l}\text { Decoction, } \\
\text { Infusion }\end{array}$ \\
\hline
\end{tabular}




\begin{tabular}{|c|c|c|c|c|c|c|c|}
\hline 35 & Piyak iyub & Oxalis corniculata $L$. & Oxalidaceae & $\begin{array}{l}\text { Ring Worm .Stons } \\
\text { Diabetes, Tonsillitis, } \\
\text { Dysentery }\end{array}$ & Herbs & Leaves & Poultices, Infusion \\
\hline 36 & Yepe tare & Paederia foetida $L$. & Rubiaceae & Dysentery, Diabetes & Climber & Leaves & Infusion \\
\hline 37 & Namdung & Perilla ocymoides $L$. & Lamiaceae & $\begin{array}{l}\text { Heart Problems, } \\
\text { Allergies Cold Anc } \\
\text { Coughs }\end{array}$ & Herb & Seed & Powder \\
\hline 38 & Pan anne & Piper betle L. & Piperaceae & $\begin{array}{l}\text { Cold And Cough, } \\
\text { Mouth Wash }\end{array}$ & Climber & Leaves & Raw, Paste \\
\hline 39 & Jalub & Piper nigrum $L$ & Piperaceae & $\begin{array}{l}\text { Cold And Cough, } \\
\text { Fever }\end{array}$ & Climber & Fruit & $\begin{array}{l}\text { Raw, Paste } \\
\text {,Infusion }\end{array}$ \\
\hline 40 & Rori & Piper pedicellatum C.DC & Piperaceae & $\begin{array}{l}\text { Constipation, } \\
\text { Stomach } \\
\text { Tonic }\end{array}$ & Herb & $\begin{array}{l}\text { Leave, } \\
\text { Fruit }\end{array}$ & Raw, Paste \\
\hline 41 & $\begin{array}{c}\text { Donyi } \\
\text { sunkang }\end{array}$ & Plantago major $L$. & Plantaginaceae & $\begin{array}{l}\text { Gastritis, Urine Flow } \\
\text { Stone, Tonic, }\end{array}$ & Herb & Leave & Decoction, Infusion \\
\hline 42 & Tapir & $\begin{array}{c}\text { Phoebe cooperiana U.N } \\
\text { Kanjilal ex A. Das }\end{array}$ & Lauraceae & Dysentery, Diarrhoea & Tree & $\begin{array}{l}\text { Fruit, } \\
\text { Leaves }\end{array}$ & Raw \\
\hline 43 & Sayong & Polygonum hydropiper $L$. & polygonaceae & Menstrual Problem, & Herb & Leaves & Decoction, Infusion \\
\hline 44 & Oyik & $\begin{array}{c}\text { Gonostegia hirta (Hassk.) } \\
\text { Miq. }\end{array}$ & Urticaceae & Stomach Ache & Herb & Leaves & Decoction \\
\hline 45 & Aki Rokmi & Ricinus communis $L$ & Euphorbiaceae & $\begin{array}{l}\text { Wound Healing, } \\
\text { Backache, Muscle } \\
\text { Ache }\end{array}$ & Shrub & Leaves & Raw, Poultice \\
\hline 46 & Gam oying & $\begin{array}{l}\text { Breynia androgyna (L.) } \\
\text { Chakrab. and N.P.Balakr }\end{array}$ & Phyllanthaceae & $\begin{array}{l}\text { Eye Tonic, Multi- } \\
\text { Vitamins. }\end{array}$ & Shrub & Leave & $\begin{array}{l}\text { Decoction, } \\
\text { Infusion }\end{array}$ \\
\hline 47 & $\begin{array}{l}\text { Tasut } \\
\text { Nekung }\end{array}$ & Smilax aspera $L$. & Smilacaceae & Itching, Rash & Climber & Fruit & Raw, Paste \\
\hline
\end{tabular}




\begin{tabular}{|c|c|c|c|c|c|c|c|}
\hline 48 & Peyomg & Scoparia dulcis $L$ & Plantaginaceae & Gastric & Herb & Leaves & $\begin{array}{l}\text { Decoction, } \\
\text { Infusion }\end{array}$ \\
\hline 49 & Donyi gori & Senna alata (L.) Roxb & Caesalpinioideae & $\begin{array}{l}\text { Fungal Infection, } \\
\text { Ringworm }\end{array}$ & Herb & Leaves & $\begin{array}{l}\text { Decoction, } \\
\text { Infusion }\end{array}$ \\
\hline 50 & Bangko & Solanum spirale Roxb. & Solanaceae & $\begin{array}{l}\text { Gastritis, Malaria anc } \\
\text { Debility }\end{array}$ & Shrub & $\begin{array}{l}\text { Leaves anc } \\
\text { Fruit }\end{array}$ & $\begin{array}{l}\text { Decoction,Infusio } \\
\mathrm{n}\end{array}$ \\
\hline 51 & Kopir & Solanum viarum Dunal & Solanaceae & $\begin{array}{l}\text { Spleen Problem. } \\
\text { Kidney Stones }\end{array}$ & Shrub & $\begin{array}{l}\text { Leaves, } \\
\text { Fruit }\end{array}$ & $\begin{array}{l}\text { Decoction, } \\
\text { Infusion }\end{array}$ \\
\hline 52 & Okomamang & Solanum nigrum $L$. & Solanaceae & $\begin{array}{l}\text { Anaemia, Liver Tonic } \\
\text { Gynaecological } \\
\text { Disorders }\end{array}$ & Herb & Leaves, Fruit & Decoction, Infusion \\
\hline 53 & Kodu & Solanum torvum $S w$. & Solanaceae & $\begin{array}{l}\text { Spleen And Stone } \\
\text { Problem }\end{array}$ & Herb, & Fruit, Seed & $\begin{array}{l}\text { Decoction, } \\
\text { Infusion }\end{array}$ \\
\hline 54 & Ogen & Sonchus oleraceus L. & Asteraceae & Stone, Gastritis & Herb & Leaves & $\begin{array}{l}\text { Decoction, } \\
\text { Infusion }\end{array}$ \\
\hline 55 & Marsang & $\begin{array}{l}\text { Acmella oleracea } \\
\text { (L.) R.K.Jansen }\end{array}$ & Asteraceae & $\begin{array}{l}\text { Cough, } \\
\text { Painkiller }\end{array}$ & Herb & Seeds, Leaves $n$ & nfusion, Raw \\
\hline 56 & $\begin{array}{c}\text { Peji- } \\
\text { pemang }\end{array}$ & Urtica urens $L$. & Urticaceae & $\begin{array}{l}\text { Headache With } \\
\text { Spleen Pains. }\end{array}$ & Herb & $\begin{array}{l}\text { Tender } \\
\text { Leave }\end{array}$ & $\begin{array}{l}\text { Decoction, } \\
\text { Infusion }\end{array}$ \\
\hline 57 & Onger & $\begin{array}{l}\text { Zanthoxylum rhetsa } \\
\text { (Roxb.) DC. }\end{array}$ & Rutaceae & $\begin{array}{l}\text { Painkiller, Cough } \\
\text {,Asthma }\end{array}$ & Shrub & $\begin{array}{l}\text { Leaves, } \\
\text { Fruits, Seeds, }\end{array}$ & Infusion \\
\hline 58 & Rekom & $\begin{array}{c}\text { Zanthoxylum } \\
\text { nitidum (Roxb.) } \\
\text { DC. }\end{array}$ & Rutaceae & $\begin{array}{l}\text { Cancer, Pain Killer } \\
\text { Asthma, }\end{array}$ & Shrub & $\begin{array}{l}\text { Seeds, Stem, } \\
\text { Leave }\end{array}$ & Infusion \\
\hline 59 & Takeeng & Zingiber officinale Roscoe & Zingiberaceae & $\begin{array}{l}\text { Cold and Cough, } \\
\text { Tonsillitis, Vomiting }\end{array}$ & Herb & Rhizome & Decoction \\
\hline
\end{tabular}



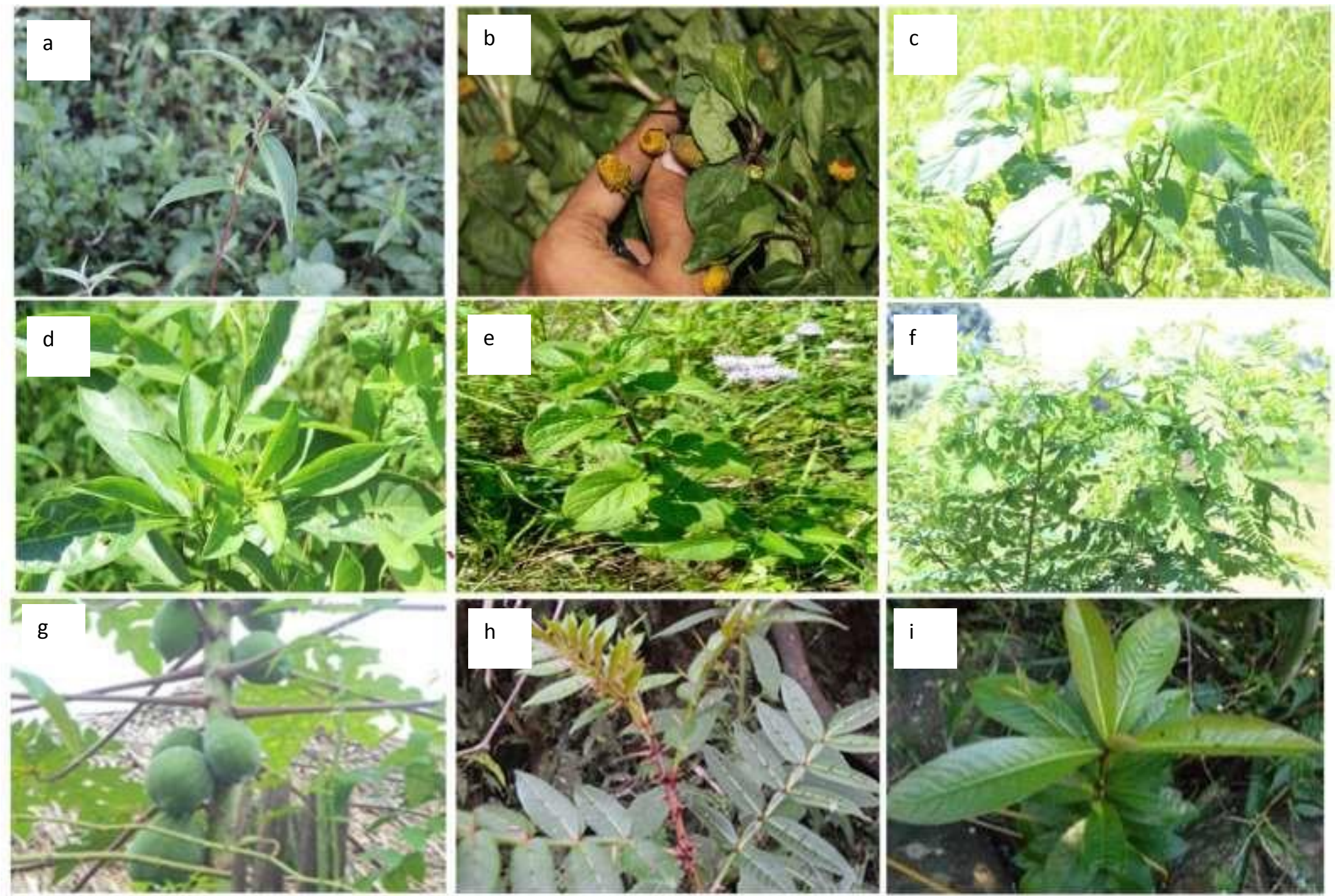

Fig. 2 - (a) Pouzolzia hirta; (b) Aemella oleracea; (b); (b) Spilanthes paniculata; (c) Clerodendron colebrookianum; (d) Solanum spirale; (e) Ageratum conyeoides; (f) Sauropus androgynus; (g) Carica papaya; (b) Zantholum rhetsa; (i) Ardisia solanacea. 


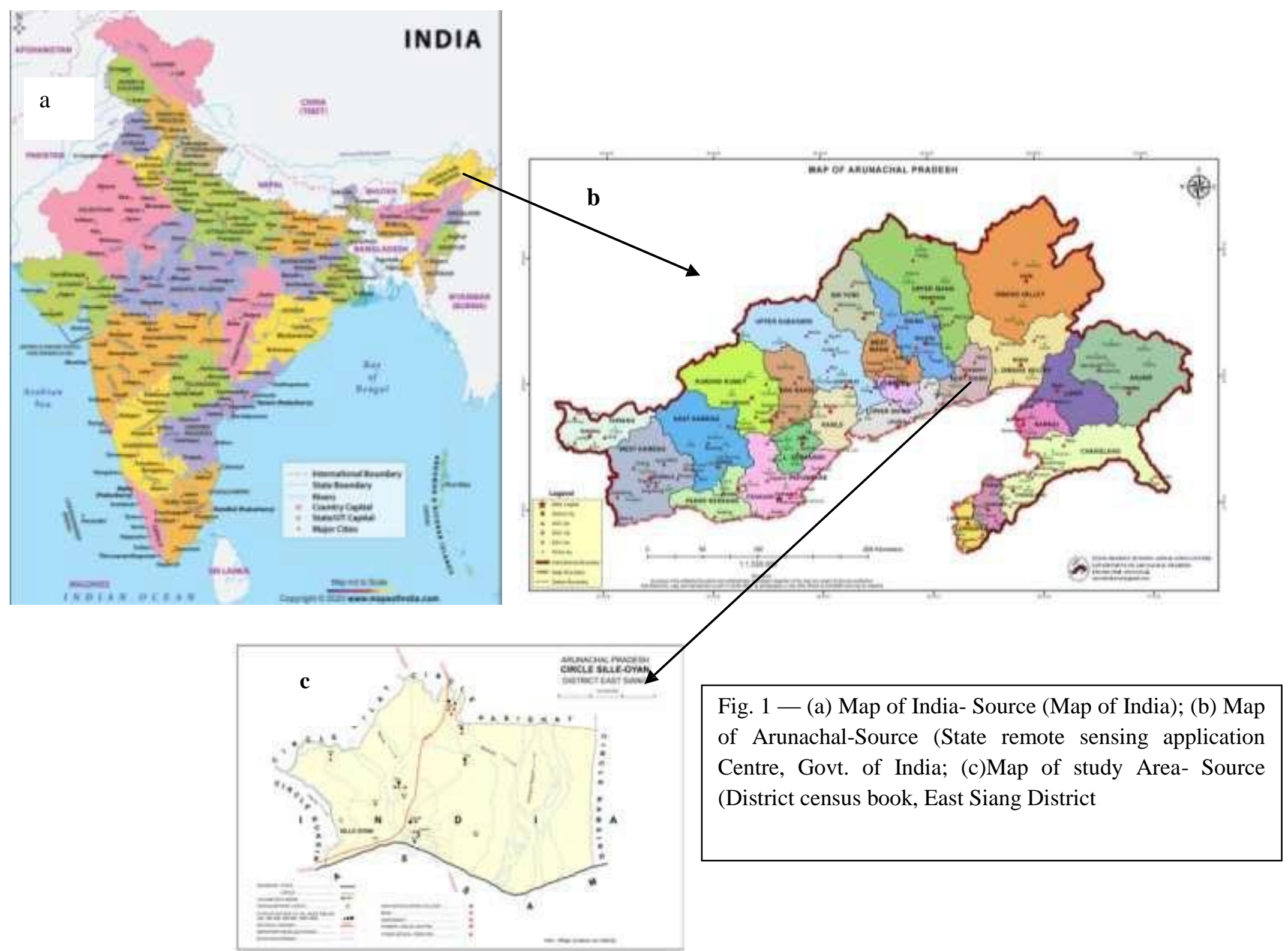


The study identified 59 plants which are used by the peoples residing in the oyan-sille circle treating ailments and has been presented in table 1. During the survey, it was found that various parts of the plants' species are used like seeds, stem, root and rhizome which belong to 34 families. Among the family Asteraeceae (6) species, solanaceae (4) were mostly used. Out of total plant species 36 herbs, 16 shrubs, 6 trees and 5 climbers. The leaves were then widely used plant parts and the method was both decoction and infusion. During the study it was found the various plants are also used in religious and most of the plants which are used in traditional medicine are used as vegetables by the villagers and the people residing in the city buy vegetables which are sell by those villagers for income generation, the plants like Clerodendron colebrookianum, Houttuynia cordata, Sauropus androgynous, Splinthus acmella etc., through which the family income of the villagers are also generated and they can improved their living standard by growing these plants as home garden.

In conclusion, traditional medicines are importance for the primary healthcare in this region since most of the village has to go far from the village to city for the modern medical facility. There is a significant aspect of studying the traditional method of medicine from which we can know the importance of the plants and in which ailments they are used by which scientific validation of plants used by local communities could be beneficial for the development of modern potential drugs. Therefore there is an urgent need of scientific screening of the plants for identifying the bioactive compounds for the formulation of new drugs. They are useful for the management, conservation and documentation of some plants which are widely exploited by which their populations in the wild are in a great risk of extinction so; they must be brought in cultivation for their availability. The state forest department, NGOs in collaborating with villager should conduct awareness program for the management, protection and cultivation of the species. They should teach them not to take out all the plant species from a particular area as these plants may be endemic to that area and if these are harvested we should leave some plant and spread seeds in that area from regeneration. The women should also play a key role in conservation and sustainable use of the biodiversity, as they are the primary collectors of the house for domestic consumption.

\section{Acknowledgements}

The authors express their gratitude to all the Adi villagers of Sille Oyan Circle who have shared their valuable knowledge. They are also thankful to Dean, College of Horticulture and Forestry, Central Agricultural University, Pasighat for his guidance and encouragement.

Conflicts: The authors declared no conflicts of interest

\section{References}

Baruah, S., Borthakur, S.K., Gogoi, P., and Ahmed, A. 2013. Ethnomedicinal plants used by Adi -Minyong tribe of Arunachal Pradesh, Eastern Himalaya. Indian J. Trad. Knowl 4(3), 278-282.

Berkes, F., 2018. Sacred ecology: Traditional ecological knowledge and resource management. New York. Taylor and Francis.

Dutta, P., and Ahmad, S.I. 1995. People of India, Arunachal Pradesh, Vol. XVI. Anthropological Survey of India, Calcutta.

Holmstedt, B., and Bruhn, J.G., 1983. Ethnopharmacology - a challenge. 
Journal of Ethnopharmacology. 8(3), 251-256.

Mandal, H., Mulherjee, S., and Datta, A. 2002. India an illustrated Atlas of Tribal World. Anthropological Survey of India, Kolkata.

Nimasow, G., Ringu, N., and Nimasow, O.D. 2012. Ethnomedicinal Knowledge Among the Adi Tribes of Lower Dibang Valley, Arunachal Pradesh, India. Intrn. Res. J. Pharm. 3(6), 223-229.

Reid, W.V., Berkes, F., Wlbanks, T., and Capistrano, D. 2006. Bridging scales and knowledge systems: linking global science and local knowledge in assessments. Millennium Ecosystem Assessment and Island, Washington.

Sharma, T.K., and Borthakur, S.K. 2008. Ethnobotanical observations on Bamboos among Adi tribes in Arunachal Pradesh. Indian J. Trad. Knowl. 7(4), $594-597$.

Singh, R.K., Singh, A., and Bhardwaj, R. 2012. Namdung (Perilla ocymoides): A Bioculturally rich plant in food and livelihood security of Adi Women in
Arunachal Pradesh, Eastern Himalaya. Indian J. Trad. Knowl. 11(1), 143 - 149.

Singh, R.K., Singh, A., Tag, H., and Adi community. 2008. Traditional Skill among the Adi tribes of Arunachal Pradesh, Indian J. Trad. Knowl. 7 (1), 27-36.

Singh, R.K., Srivastava, R.C., Adi community and Mukherjee, T.K. 2010. Toko-Patta (Liviston jenkinsiana Griff): Adi community and conservation of culturally important endangered tree species in eastern Himalaya. Indian J. Trad. Knowl, 9(2), 231-241

Singh, S.K., 1998. India's Communities A-G. Vol. IV. Anthropological Survey of India, Calcutta.

World Health Organization.WHO Traditional Medicine Strategy2002-2005.Geneva. http://www.who.int/medicines/library/tr $\mathrm{m} / \mathrm{trm} \_$str at_eng.pdf.

Yumnum, J.Y., and Tripathi, O.P., 2013. Ethnobotany: Plants use in fishing and hunting by Adi Tribe of Arunachal Pradesh. Indian J. Trad. Knowl. 12(1), 15-16.

\section{How to cite this article:}

Tantulung Tatan, T.S. Mehra, Senjem Semba, Haffis Mohammed and Fullmoon Puwein. 2020. Plants Used as Ethanomedicine by the Adi Tribes of Sille-Oyan Circle of Arunachal Pradesh: Source for Livelihood and Medicine. Int.J.Curr.Microbiol.App.Sci. 9(12): 2941-2951. doi: https://doi.org/10.20546/ijcmas.2020.912.349 